SLAC-PUB-11511

SU-ITP-05/29

\title{
Complex/Symplectic Mirrors
}

\author{
Wu-yen Chuang ${ }^{1,2}$, Shamit Kachru ${ }^{1,2}$ and Alessandro Tomasiello ${ }^{1}$ \\ ${ }^{1}$ ITP, Stanford University, Stanford, CA 94305, USA \\ ${ }^{2}$ SLAC, Stanford University, Menlo Park, CA 94309, USA
}

\begin{abstract}
We construct a class of symplectic non-Kähler and complex non-Kähler string theory vacua, extending and providing evidence for an earlier suggestion by Polchinski and Strominger. The class admits a mirror pairing by construction. Comparing hints from a variety of sources, including ten-dimensional supergravity and KK reduction on $\mathrm{SU}(3)-$ structure manifolds, suggests a picture in which string theory extends Reid's fantasy to connect classes of both complex non-Kähler and symplectic non-Kähler manifolds.
\end{abstract}

\section{Complex and symplectic vacua}

The study of string theory on Calabi-Yau manifolds has provided both the most popular vacua of the theory, and some of the best tests of theoretical ideas about its dynamics. Most manifolds, of course, are not Calabi-Yau. What is the next simplest class for theorists to explore?

The answer, obviously, depends on what the definition of "simplest" is. However, many leads seem to be pointing to the same suspects. First of all, it has been suggested long ago [1] that type II vacua exist, preserving $\mathcal{N}=2$ supersymmetry (the same as for Calabi-Yau's), on manifolds which are complex and non-Kähler (and enjoy vanishing $c_{1}$ ). Calabi-Yau manifolds are simultaneously complex and symplectic, and mirror symmetry can be viewed as an exchange of these two properties [2]. The same logic seems to suggest that the proposal of [1] should also include symplectic non-Kähler manifolds as mirrors of the complex non-Kähler ones. Attempts at providing mirrors of this type (without using a physical interpretation) have indeed already been made in [3, 4. 
In a different direction, complex non-Kähler manifolds have also featured in supersymmetry-preserving vacua of supergravity, already in [5]. More recently the general conditions for preserving $\mathcal{N}=1$ supersymmetry in supergravity have been reduced to geometrical conditions [6]; in particular, the manifold has to be generalized complex [7]. The most prominent examples of generalized complex manifolds are complex and symplectic manifolds, neither necessarily Kähler. It should also be noted that complex and symplectic manifolds seem to be natural in topological strings.

In this paper we tie these ideas together. We find that vacua of the type described in [1] can be found for a large class of complex non-Kähler manifolds in type IIB and symplectic non-Kähler manifolds in type IIA, and observe that these vacua come in mirror pairs. Although these vacua are not fully amenable to ten-dimensional supergravity analysis for reasons that we will explain (this despite the fact that they preserve $\mathcal{N}=2$ rather than $\mathcal{N}=1$ supersymmetry), this is in agreement with the supergravity picture that all (RR) $\mathrm{SU}(3)$-structure IIA vacua are symplectic [8], and all IIB vacua are complex [9, 10, 8, possibly suggesting a deeper structure.

In section 2, in an analysis formally identical to [1], we argue for the existence of the new vacua. In section 3 we show that the corresponding internal manifolds are not Calabi-Yau but rather complex or symplectic. More specifically, in both theories, they are obtained from a transition that does not preserve the Calabi-Yau property. As evidence for this, we show that the expected physical spectrum agrees with the one obtained on the proposed manifolds. The part of this check that concerns the massless spectrum is straightforward; we can extend it to low-lying massive fields by combining results from geometry [11] and KK reduction on manifolds of $\mathrm{SU}(3)$ structure. We actually try in section 4 to infer from our class of examples a few properties which should give more control over this kind of KK reduction. Specifically, we suggest that the lightest massive fields should be in correspondence with pseudo-holomorphic curves or pseudo-SpecialLagrangian three-cycles (a notion we will define at the appropriate juncture).

Among the motivations for this paper were also a number of more grandiose questions about the effective potential of string theory. One of the motivations for mathematicians to study the generalized type of transition we consider in this paper is the hope that many moduli spaces actually happen to be submanifolds of a bigger moduli space, not unlike 12 the realization of the various 19-dimensional moduli spaces of algebraic K3's as submanifolds of the 20-dimensional moduli space of abstract K3's. It might be that 
string theory provides a natural candidate for such a space, at least for the $\mathcal{N}=2$ theories, whose points would be all SU(3)-structure manifolds (not necessarily complex or symplectic), very possibly augmented by non-geometrical points [13]. We would not call it a moduli space, but rather a configuration space: on it, a potential would be defined, whose zero locus would then be the moduli space of $\mathcal{N}=2$ supersymmetric string theory vacua, including in particular the complex and symplectic vacua described here. In this context, what this paper is studying is a small neighborhood where the moduli space of $\mathcal{N}=2$ non-Kähler compactifications meets up with the moduli space of Calabi-Yau compactifications with RR flux, inside this bigger configuration space of manifolds.

\section{Four-dimensional description of the vacua}

We will now adapt the ideas from [1] to our needs. The strategy is as follows. We begin by compactifying the IIB and IIA strings on Calabi-Yau threefolds, and we switch on internal RR fluxes, $F_{3}$ in IIB and $F_{4}$ in IIA (our eventual interest will be the case where the theories are compactified on mirror manifolds $\mathcal{M}$ and $\mathcal{W}$, and the fluxes are mirror to one another). As also first noted in [1], this will make the four-dimensional $\mathcal{N}=2$ supergravity gauged; in particular, it will create a potential on the moduli space. This potential has supersymmetric vacua only at points where the Calabi-Yau is singular. However, on those loci of the moduli space new massless brane hypermultiplets have to be taken into account, which will then produce the new vacua.

\subsection{The singularities we consider}

Let us first be more precise about the types of singularities we will consider. In IIB, as we will review shortly, if we switch on $F_{3}$ with a non-zero integral along a cycle $B_{3}$ of a Calabi-Yau $\mathcal{M}$, a supersymmetric vacuum will exist on a point in moduli space in which only the cycle $A_{3}$ conjugate to $B_{3}$ under intersection pairing shrinks. It is often the case that several cycles shrink simultaneously, with effects that we will review in the next section, but there are definitely examples in which a single $B$ cycle shrinks. These are the cases we will be interested in. (We will briefly explain in section 3.2 how this condition could be relaxed.)

In IIA, switching on $F_{4}$ with a non-zero integral on a four-cycle $\tilde{A}_{4}$ of $\mathcal{W}$ will generate 
a potential which will be zero only in points in which the quantum-corrected volume of the conjugate two-cycle $\tilde{B}_{2}$ (the Poincaré dual to $F_{4}$ ) vanishes. This will happen on a wall between two birationally equivalent Calabi-Yau's, connected by a flop of $\tilde{B}_{2}$. These points will be mirror to the ones we described above for IIB.

The converse is not always true: there can be shrinking three-cycles which are mirror to points in the IIA moduli space in which the quantum volume of the whole Calabi-Yau goes to zero. These walls separate geometrical and Landau-Ginzburg, or, hybrid, phases. One would obtain a vacuum at such a point by switching on $F_{0}$ instead of $F_{4}$, for instance. The example discussed in [1] (the quintic) is precisely such a case. Since in the end we want to give geometrical interpretations to the vacua we will obtain, we will restrict our attention only to cases in which a curve shrinks in $\mathcal{W}$ - that is, when a flop happens. Although this is not strictly necessary for IIB, keeping mirror symmetry in mind we will restrict our attention to cases in which the stricter IIA condition is valid, not only the IIB one: in the mirror pairs of interest to us, the conifold singularity in $\mathcal{M}$ is mirror to a flop in $\mathcal{W}$. It would be interesting, of course, to find the IIA mirrors to all the other complex non-Kähler manifolds in IIB.

Looking for flops is not too difficult, as there is a general strategy. If the Calabi-Yau $\mathcal{W}$ is realized as hypersurface in a toric manifold $V$, the "enlarged Kähler moduli space" [14, 15] (or at least, the part of it which comes from pull-back of moduli of $V$ ) is a toric manifold $W_{K}$ itself. The cones of the fan of $W_{K}$ are described by different triangulations of the cone over the toric polyhedron of $V$. Each of these cones will be a phase [16]; there will be many non-geometrical phases (Landau-Ginzburg or hybrid). Fortunately, the geometrical ones are characterized as the triangulations of the toric polyhedron of $V$ itself (as opposed to triangulations of the cone over it). This subset of cones gives an open set in $W_{K}$ which is called the "partially enlarged" Kähler moduli space. This is not the end of the story, however. In many examples, it will happen that a flop between two geometrical phases will involve more than one curve at a time, an effect due to restriction from $V$ to $\mathcal{W}$. Worse still, these curves might have relations, and sometimes there is no quick way to determine this. Even so, we expect that there should be many cases in which a single curve shrinks (or many, but without relations).

Such an example is readily found in the literature [17, 18]: taking $\mathcal{W}$ to be an elliptic fibration over $\mathbb{F}_{1}$ (a Calabi-Yau whose Hodge numbers are $h^{1,1}=3$ and $h^{2,1}=243$ ), there is a point in moduli space in which a single curve shrinks (see Appendix $\mathrm{A}$ for 
more details). By counting of multiplets and mirror symmetry, on the mirror $\mathcal{M}$ there will be a single three-cycle which will shrink. This implies that the mirror singularity will be a conifold singularity. Indeed, it is a hypersurface singularity, and as such the shrinking cycle is classified by the so-called Milnor number. This has to be one if there is a single shrinking cycle, and the only hypersurface singularity with Milnor number one is the conifold.

\subsection{Gauged supergravity analysis}

After these generalities, we will now show how turning on fluxes drives the theory to a conifold point in the moduli space; more importantly, we will then show how including the new massless hypermultiplets generates new vacua. We will do this in detail in the IIB theory on $\mathcal{M}$, as its IIA counterpart is then straightforward. The analysis is formally identical to the one in [1] (see also [19, 20]); the differences have been explained in the previous subsection.

As usual, define the symplectic basis of three-cycles $A^{I}, B_{J}$ and their Poincaré duals $\alpha_{I}, \beta^{I}$ such that

$$
A^{I} \cdot B_{J}=\delta_{J}^{I}, \quad \int_{A^{J}} \alpha_{I}=\int_{B_{I}} \beta^{J}=\delta_{I}^{J}
$$

along with the periods $X^{I}=\int_{A^{I}} \Omega$ and $F_{I}=\int_{B_{I}} \Omega$. Additionally, the basis is taken so that the cycle of interest described in subsection 2.1 is $A=A^{1}$.

When $X^{1}=0$, the cycle $A^{1}$ degenerates to the zero size and $\mathcal{M}$ develops a conifold singularity. By the monodromy argument, the symplectic basis $\left(X^{1}, F_{1}\right)$ will transform as follows when we circle the discriminant locus in the complex moduli space defined by $X^{1}=0$ :

$$
X^{1} \rightarrow X^{1} \quad F_{1} \rightarrow F_{1}+X^{1} .
$$

From this we know $F_{1}$ near the singularity:

$$
F_{1}=\text { constant }+\frac{1}{2 \pi i} X^{1} \ln X^{1}+\ldots
$$

The metric on the moduli space can be calculated from the formulae

$$
\mathcal{G}_{I \bar{J}}=\partial_{I} \partial_{\bar{J}} K_{V}, \quad K_{V}=-\ln i\left(\bar{X}^{I} F_{I}-X_{I} \bar{F}^{I}\right) .
$$

Therefore we obtain

$$
\mathcal{G}_{1 \overline{1}} \sim \ln \left(X^{1} \bar{X}^{1}\right)
$$


Now, the internal flux we want to switch on is $F_{3}=n_{1} \beta^{1}$. The vectors come from

$$
F_{5}=F_{2}^{I} \wedge \alpha_{I}-G_{2, I} \wedge \beta^{I},
$$

where the $F_{2}^{I}\left(G_{2 I}\right)$ is the electric (magnetic) field strength. The Chern-Simons coupling in the IIB supergravity action is then

$$
\epsilon^{i j} \int_{M_{4} \times C Y} \tilde{F}_{5} \wedge H_{3}^{i} \wedge B_{2}^{j}=n_{1} \int_{M_{4}} F_{2}^{1} \wedge B_{2}
$$

where $M_{4}$ is the spacetime. By integration by parts, and since $B_{2}$ dualizes to one of the (pseudo)scalars in the universal hypermultiplets, we see that the latter is gauged under the field $A^{1}$ whose field strength is $d A^{1}=F_{2}^{1}$.

The potential is now given by the "electric" formula

$$
V=h_{u v} k_{I}^{u} k_{J}^{v} \bar{X}^{I} X^{J} e^{K_{V}}+\left(U^{I J}-3 \bar{X}^{I} X^{J} e^{K_{V}}\right) \mathcal{P}_{I}^{\alpha} \mathcal{P}_{J}^{\alpha}
$$

where

$$
U^{I J}=D_{a} X^{I} g^{a \bar{b}} D_{\bar{b}} X^{J}
$$

and the $\mathcal{P}^{\alpha}$ are together the so-called Killing prepotential, or hypermomentum map. In our situation only the flux over $B_{1}$ is turned on, and the Killing prepotential is given by

$$
\mathcal{P}_{1}^{1}=\mathcal{P}_{1}^{2}=0 ; \quad \mathcal{P}_{1}^{3}=-e^{\tilde{K}_{H}} n_{e I}^{(2)}=-e^{2 \phi} n_{e I}^{(2)}
$$

where $\phi$ is the dilaton. The potential will then only depend on the period of the dual $A^{1}$ cycle, call it $X^{1}$ :

$$
V \sim \frac{\left(n_{1}\right)^{2}}{\ln X^{1} \bar{X}^{1}} .
$$

The theory will thus be driven to the conifold point where $X^{1}=0$.

This is not the end of the story: at the singular point, one has a new massless hypermultiplet $B$ coming from a brane wrapping the shrinking cycle $A^{1}$. The world-volume coupling between the D3-brane and $F_{5}$ gives then $\int_{\mathbb{R} \times A^{1}} A_{4}=\int_{\mathbb{R}} A^{1}$, where $\mathbb{R}$ is the worldline of the resulting light particle in $M_{4}$. (The coincidence between the notation for the cycle $A^{1}$ and the corresponding vector potential $A^{1}$ is rather unfortunate, if standard.)

This means that both the universal and the brane hypermultiplet are charged under the same vector; we can then say that they are all electrically charged and still use 
the electric formula for the potential (2.8), with the only change being that the Killing prepotential is modified to be

$$
\mathcal{P}_{1}^{\alpha}=\left.\mathcal{P}_{1}^{\alpha}\right|_{B=0}+B^{+} \sigma^{\alpha} B
$$

the black hole hypermultiplet is an $S U(2)$ doublet with components $\left(B_{1}, B_{2}\right)$. Loci on which the $\mathcal{P}^{\alpha}$ 's are zero are new vacua: it is easy to see that they are given by

$$
B=\left(\left(e^{\tilde{K}_{H}} n_{1}\right)^{1 / 2}, 0\right)=\left(e^{\phi} n_{1}^{1 / 2}, 0\right) .
$$

The situation here is similar to [1]: the expectation value of the new brane hypermultiplet is of the order $g_{s}=e^{\phi}$. So, as in that paper, the two requirements that $g_{s}$ is small and that $B$ be small (the expression for the $\mathcal{P}^{\alpha}$ is a Taylor expansion and will be modified for large $B$ ) coincide, and with these choices we can trust these vacua. After the Higgsing the flat direction of the potential, namely, the massless hypermultiplet $\tilde{B}_{0}$, would be a linear combination of the brane hypermultiplet and the universal hypermultiplet while the other combination would become a massive one $\tilde{B}_{m}$.

\subsection{The field theory capturing the transition}

It is useful to understand the physics of the transition from a $4 \mathrm{~d}$ field theory perspective, in a region very close to the transition point on moduli space. While this analysis is in principle a simple limit of the gauged supergravity in the previous subsection, going through it will both provide more intuition and also allow us to infer some additional lessons. In fact, in the IIB theory with $n_{1}$ units of RR flux, the theory close to the transition point (focusing on the relevant degrees of freedom) is simply a $U(1)$ gauge theory with two charged hypers, of charges 1 and $n_{1}$.

Let us focus on the case $n_{1}=1$ for concreteness. Let us call the $\mathcal{N}=1$ chiral multiplets in the two hypers $B, \tilde{B}$ and $C, \tilde{C}$. In $\mathcal{N}=1$ language, this theory has a superpotential

$$
W \sim \tilde{B} \varphi B+\tilde{C} \varphi C
$$

where $\varphi$ is the neutral chiral multiplet in the $\mathcal{N}=2 \mathrm{U}(1)$ vector multiplet. It also has a D-term potential

$$
|D|^{2} \sim\left(|\tilde{B}|^{2}-|B|^{2}+|\tilde{C}|^{2}-|C|^{2}\right)^{2}
$$


There are two branches of the moduli space of vacua: a Coulomb branch where $\langle\varphi\rangle \neq 0$ and the charged matter fields vanish, and a Higgs branch where $\langle\varphi\rangle=0$ and the hypers have non-vanishing vevs (consistent with $F$ and $D$ flatness). The first branch has complex dimension one, the second has quaternionic dimension one. These branches meet at the point where all fields have vanishing expectation value.

At this point, the theory has an $\mathrm{SU}(2)$ global flavor symmetry. This implies that locally, the hypermultiplet moduli space will take the form $\mathbb{C}^{2} / \mathbb{Z}_{2}[21$. In fact, the precise geometry of the hypermultiplet moduli space, including quantum corrections, can then be determined by a variety of arguments [21, 22] (another type of argument 23] implies the same result for the case where the hypermultiplets coming from shrinking three-cycles in IIB). The result is the following. Locally, the quaternionic space reduces to a hyperKähler manifold which is an elliptic fibration, with fiber coordinates $t, x$ and a (complex) base coordinate $z$. Let us denote the Kähler class of the elliptic fiber by $\lambda^{2}$. Then, the metric takes the form

$$
d s^{2}=\lambda^{2}\left(V^{-1}(d t-\mathbf{A} \cdot \mathbf{d y})^{2}+V(\mathbf{d y})^{2}\right)
$$

where $\mathbf{y}$ is the three-vector with components $\left(x, \frac{z}{\lambda}, \frac{\bar{z}}{\lambda}\right)$. Here, the function $V$ and the vector of functions $\mathbf{A}$ are given by

$$
V=\frac{1}{2 \pi} \sum_{n=-\infty}^{\infty}\left(\frac{1}{\sqrt{(x-n)^{2}+\frac{|z|^{2}}{\lambda^{2}}}}-\frac{1}{|n|}\right)+\text { constant }
$$

and

$$
\nabla \times \mathbf{A}=\nabla V .
$$

This provides us with detailed knowledge of the metric on the hypermultiplet moduli space emanating from the singularity, though it is hard to explicitly map the flat direction to a combination of the universal hypermultiplet and the geometrical parameters of $\mathcal{M}^{\prime}$ or $\mathcal{W}^{\prime}$. We shall discuss some qualitative aspects of this map in $\S 3.3$. For the reader who is confused by the existence of a Coulomb branch at all, given that e.g. in the IIB picture $F_{3} \neq 0$, we note that the Coulomb branch will clearly exist on a locus where $g_{s} \rightarrow 0$ (since the hypermultiplet vevs must vanish). This is consistent with supergravity intuition, since in the $4 \mathrm{~d}$ Einstein frame, the energetic cost of the RR fluxes vanishes as $g_{s} \rightarrow 0$. 


\section{Geometry of the vacua}

We will first of all show that the vacua obtained in the previous section cannot come from a transition to another Calabi-Yau. To this aim, in the next subsection we will review Calabi-Yau extremal transitions. We will then proceed in subsection 3.2 to review the less well-known non-Calabi-Yau extremal transitions, and then compare them to the vacua we previously found in subsection 3.3 .

\subsection{Calabi-Yau extremal transitions}

Calabi-Yau extremal transitions sew together moduli spaces for Calabi-Yaus whose Hodge numbers differ; let us quickly review how. For more details on this physically well--studied case, the reader might want to consult [24, 25, 26, 14].

Consider IIB theory on a Calabi-Yau $\mathcal{M}$. (Some of the explanations in this paper are given in the IIB case only, whenever the IIA case would be an obvious enough modification). Suppose that at a particular point in moduli space, $\mathcal{M}$ develops $N$ nodes (conifold points) by shrinking as many three-cycles $A_{a}, a=1, \ldots, N$, and that these three-cycles satisfy $R$ relations

$$
\sum_{a=1}^{N} r_{i}^{a} A_{a}=0, \quad i=1, \cdots, R
$$

in $H_{3}$. We are not using the same notation for the index on the cycles as in section 2, as these $A_{a}$ are not all elements of a basis (as they are linearly dependent). Notice that it is already evident that this case is precisely the one we excluded with the specifications in section 2.1. To give a classic example [24, there is a known transition where $\mathcal{M}$ is the quintic, $N=16$ and $R=1$. Physically, there will be $N$ brane hypermultiplets $B_{a}$ becoming massless at this point in moduli space. Vectors come from $h^{2,1}$; since the $B_{a}$ only span $N-R$ directions in $H^{3}$, they will be charged under $N-R$ vectors $X^{A}$ only, $A=1, \ldots N-R$. Call the matrix of charges $Q_{A}^{a}, A=1, \ldots, N-R, a=1, \ldots N$.

In this case, when looking for vacua, we will still be setting the Killing prepotential $\mathcal{P}_{a}$ (which is a simple extension of the one in (2.12) ) to zero: the flux is now absent, and the $B^{2}$ term now reads

$$
\mathcal{P}_{A}=\sum_{a} Q_{A}^{a} B_{a}^{+} \sigma^{\alpha} B_{a}
$$

Notice that we have switched no flux on in this case; crucially, $\mathcal{P}=0$ now will have an 
$R$-dimensional space of solutions, due to the relations.

Let us suppose this new branch is actually the moduli space for a new Calabi-Yau. This new manifold would have $h^{2,1}-(N-R)$ vectors, because all the $X^{A}$ have been Higgsed; and $h^{1,1}+R$ hypers, because of the $N B_{a}$, only $R$ flat directions have survived.

This is exactly the same result one would get from a small resolution of all the $N$ nodes. Indeed, let us call the Calabi-Yau resulting from such a procedure $\mathcal{M}^{\prime}$, and let us compute its Betti numbers. It is actually simpler to first consider a case in which a single three-cycle undergoes surgery ${ }^{1}$, which is the case without relations specified in section 2.1. we will go back to the Calabi-Yau case, in which relations are necessary, momentarily.

The result of this single surgery along a three-cycle is that $H^{3} \rightarrow H^{3}-2, H^{2} \rightarrow H^{2}$. This might be a bit surprising: one is used to think that an extremal transition replaces a three-cycle by a two-cycle. But this intuition comes from the noncompact case, in which indeed it holds. In the compact case, when we perform a surgery along a three-cycle, we really are also losing its conjugate under Poincaré pairing; and we gain no two-cycle. The difference is illustrated in a low-dimensional analogue in figure 1, in which $H^{2}$ and $H^{3}$ are replaced by $H^{0}$ and $H^{1}$.

Coming back to the Calabi-Yau case of interest in this subsection, let us now consider $N$ shrinking three-cycles with $R$ relations. First of all $H^{3}$ only changes by $2(N-R)$, because this is the number of independent cycles we are losing. But this is not the only effect on the homology. A relation can be viewed as a four-chain $F$ whose boundary is $\sum A_{a}$. After surgery, the boundary of $F$ by definition shrinks to points; hence $F$ becomes a four-cycle in its own right. This gives $R$ new elements in $H_{4}$ (or equivalently, in $H^{2}$ ). The change in homology is summarized in Table 1, along with the IIA case and, more importantly, in a more general context that we will explain. By comparing with the physical counting above, we find evidence that the new branches of the moduli space correspond to new Calabi-Yau manifolds obtained by extremal transitions.

To summarize, Calabi-Yau extremal transitions are possible without fluxes, but they require relations among the shrinking cycles. This is to be contrasted with the vacua in the previous section, where there are no relations among the shrinking cycles to provide flat directions. Instead, the flux (and resulting gauging) lifts the old Calabi-Yau moduli

\footnotetext{
${ }^{1}$ This is a purely topological computation; in a topological context, an extremal transition is called a surgery, and we will use this term when we want to emphasize we are considering purely the topology of the manifolds involved.
} 


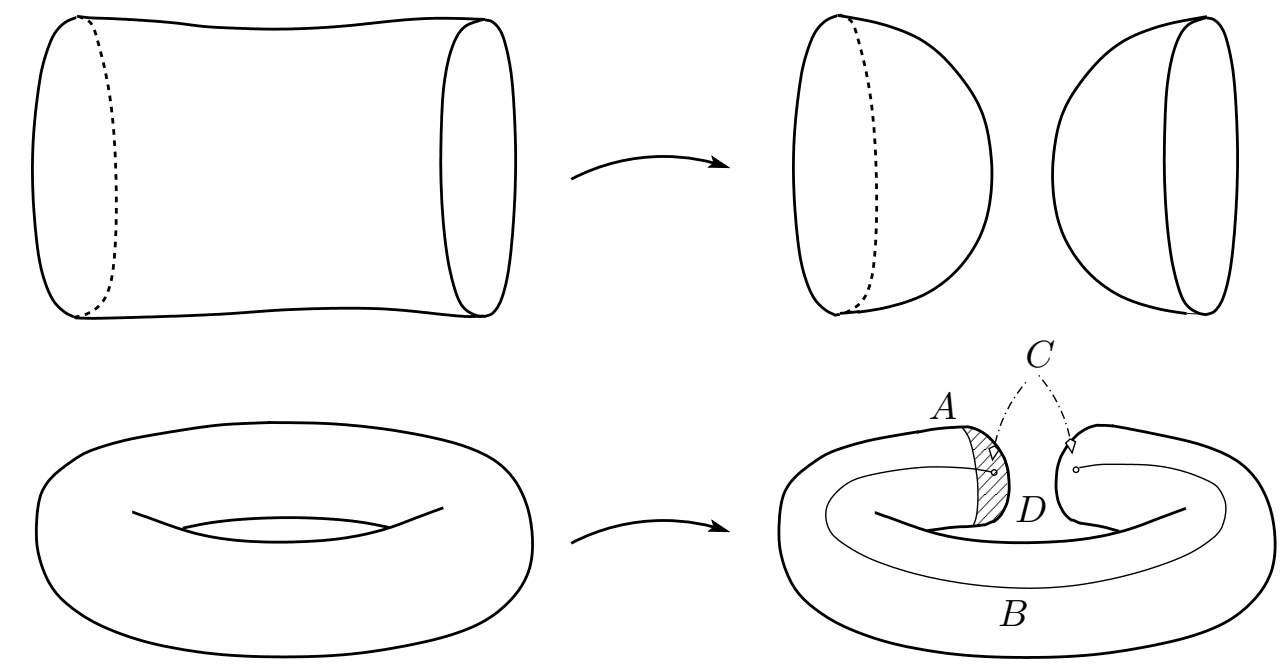

Figure 1: Difference between compact and non-compact surgery: in the noncompact case (up), one loses an element in $H^{1}$ and one gains an element in $H^{0}$ (a connected component). In the compact case (down), one loses an element in $H^{1}$ again, but the would-be new element in $H^{0}$ is actually trivial, so $H^{0}$ remains the same. This figure is meant to help intuition about the conifold transition in dimension 6 , where $H^{0}$ and $H^{1}$ are replaced by $H^{2}$ and $H^{3}$. We also have depicted various chains on the result of the compact transition, for later use.

space (as long as $g_{s} \neq 0$ ), but makes up for this by producing a new branch of moduli space (emanating from the conifold point or its mirror).

\subsection{Non-Calabi-Yau extremal transitions}

In this section we will waive the Calabi-Yau condition to reproduce the vacua of the previous section. This is, remember, a case in which cycles shrink without relations. However, we will start with a review of results in the more general case, to put in perspective both the case we will eventually consider and the usual Calabi-Yau case.

We will consider both usual conifold transitions, in which three-cycles are shrunk and replaced by curves, and so-called reverse conifold transitions, in which the converse happens. ${ }^{2}$ As a hopefully useful shorthand, we will call the first type a $3 \rightarrow 2$ transition

\footnotetext{
${ }^{2}$ Implicit in the use of the word "conifold" is the assumption that several cycles do not collapse together in a single point of the manifold $\mathcal{M}$. More general cases are also interesting to consider, see for example 26] for the complex case and [4] for the symplectic case.
} 
and the second a $2 \rightarrow 3$. Though the manifolds will no longer be (necessarily) Calabi-Yau, we will still call the initial and final manifold $\mathcal{M}$ and $\mathcal{M}^{\prime}$ in the $3 \rightarrow 2$ case (which is relevant for our IIB picture), and $\mathcal{W}$ and $\mathcal{W}^{\prime}$ in the $2 \rightarrow 3$ case (which is relevant for our IIA picture).

We will first ask whether a $3 \rightarrow 2$ transition takes a complex, or symplectic, $\mathcal{M}$ into a complex, or symplectic, $\mathcal{M}^{\prime}$, and then turn to the same questions about $\mathcal{W}, \mathcal{W}^{\prime}$ for $2 \rightarrow 3$ transitions. These questions have to be phrased a bit more precisely, and we will do so case by case.

It is also useful to recall at this point the definitions of symplectic and complex manifolds, which we will do by embedding them in a bigger framework. In both cases, we can start with a weaker concept called $G$-structure. By this we mean the possibility of taking the transition functions on the tangent bundle of $\mathcal{M}$ to be in a group $G$. This is typically accomplished by finding a geometrical object (a tensor, or a spinor) whose stabilizer is precisely $G$. If we find a two-form $J$ such that $J \wedge J \wedge J$ is nowhere zero, it gives an $\operatorname{Sp}(6, \mathbb{R})$ structure. In presence of a tensor $(1,1)$ tensor (one index up and one down) $j$ such that $j^{2}=-1$ (an almost complex structure), we speak of a $\operatorname{Gl}(3, \mathbb{C})$ structure. For us the presence of both will be important; but we also impose a compatibility condition, which says that the tensor $j_{m}^{p} J_{p n}$ is symmetric and of positive signature. This tensor is then nothing but a Riemannian metric. The triple is an almost hermitian metric: this gives a structure $\mathrm{Sp}(6, \mathbb{R}) \cap \mathrm{Gl}(3, \mathbb{R})=\mathrm{U}(3)$.

By themselves, these reductions of structure do not give much of a restriction on the manifold. But in all these cases we can now consider an appropriate integrability condition, a differential equation which makes the manifold with the given structure more rigid. In the case of $J$, we can impose that $d J=0$. In this case we say that the manifold is symplectic. For $j$, a more complicated condition (that we will detail later, when considering $\mathrm{SU}(3)$ structures) leads to complex manifolds.

Let us now consider a complex manifold $\mathcal{M}$ (which we will also take to have trivial canonical class $K=0)$. First order complex deformations are parameterized by $H^{1}(\mathcal{M}, T)=H^{2,1}$. Suppose that for some value of the complex moduli $N$ three-cycles shrink. Replace now these $N$ nodes by small resolutions. The definition of small resolution, just like the one of blowup, can be given locally around the node and then patched without any problem with the rest of the manifold. So the new manifold $\mathcal{M}^{\prime}$ is still complex. Also, the canonical class $K$ is not modified by the transition because a small 
resolution does not create a new divisor, only a new curve. ${ }^{3}$ Actually, the conjecture that all Calabi-Yau are connected was initially formulated by Reid [12 for all complex manifolds (and not only Calabi-Yaus) with $K=0$, extending ideas by Hirzebruch [27.

If now we consider a symplectic $\mathcal{M}$, the story is different. For one thing, now symplectic moduli are given by $H^{2}(\mathcal{M}, \mathbb{R})[28$, so it does not seem promising to look for a point in moduli space where three-cycles shrink. But 2.1 in 3 shows that we can nevertheless shrink a three-cycle symplectically, and replace it by a two-cycle. Whether the resulting $\mathcal{M}^{\prime}$ will also be symplectic is not automatic, however. This can be decided using Theorem 2.9 in [3]: the answer is yes precisely when there is at least one relation in homology among the three-cycles. ${ }^{4}$

The case of interest in this paper is actually a blending of the two questions considered so far, whether complex or symplectic properties are preserved. In IIB, we will take a Calabi-Yau $\mathcal{M}$ (which has both properties) and follow it in moduli space to a point at which it develops a conifold singularity. Now we perform a small resolution to obtain a manifold $\mathcal{M}^{\prime}$ and ask whether this new manifold is still Kähler; this question has been considered also by [31]. As we have seen, the complex property is kept, and the symplectic property is not (though the question in [3] regards more generally symplectic manifolds, disregarding the complex structure, and in particular being more interesting without such a path in complex structure moduli space).

Let us see why $\mathcal{M}^{\prime}$ cannot be Kähler in our case. A first argument is not too different from an argument given after figure 1 to count four-cycles. If the manifold $\mathcal{M}^{\prime}$ after the transition is Kähler, there will be an element $\omega \in H_{4}$ dual to the Kähler form. This will have non-zero intersection $\omega \cdot C_{a}=\operatorname{vol}\left(C_{a}\right)$ with all the curves $C_{a}$ produced by the small resolutions. Before the transition, then, in $\mathcal{M}, \omega$ will develop a boundary, since the $C_{a}$ are replaced by three-cycles $A_{a}$; more precisely, $\partial \omega=\sum r^{a} A_{a}$ for some coefficients $r^{a}$.

\footnotetext{
${ }^{3}$ The conditions for $\mathcal{N}=1$-preserving vacua in ten-dimensional type II supergravity actually only require $c_{1}=0$. The role of this condition is less clear for example in the topological string: for the A model it would seem to unnecessary, as there is no anomaly to cancel; for the B model, it would look like the stronger condition $K=0$ is required, which means that the canonical bundle should be trivial even holomorphically.

${ }^{4}$ We should add that the relations must involve all the three-cycles. If there is one three-cycle $A$ which is not involved in any relation, it is possible to resolve symplectically all the other cycles but not A. Examples of this type are found in [30, 31] when $\mathcal{M}$ is Kähler, which is the case of interest to us and to which we will turn shortly. These examples would play in our favor, allowing us to find even more examples of non-Kähler $\mathcal{M}^{\prime}$, but for simplicity of exposition we will mostly ignore them in the following.
} 
This proves there will have to be at least one relation between the collapsing three-cycles.

We can rephrase this in yet another way. Let us consider the case in which only one nontrivial three-cycle $A$ is shrinking. Since, as remarked earlier (see figure 1), in the compact case the curve $C$ created by the transition is trivial in homology, there exists a three-chain $B$ such that $C=\partial B$; then we have, if $J$ is the two-form of the $\mathrm{SU}(3)$ structure,

$$
0 \neq \int_{C} J=\int_{B} d J
$$

Hence $d J \neq 0$ : the manifold cannot be symplectic. ${ }^{5}$

Even if a symplectic $J$ fails to exist, there is actually a non-degenerate $J$ compatible with $j$ (since the inclusion $\mathrm{U}(3) \subset \mathrm{Sp}(6, \mathbb{R})$ is a homotopy equivalence, not unlike the way the homotopy equivalence $\mathrm{O}(n) \subset \operatorname{Gl}(n)$ allows one to find a Riemannian metric on any manifold). In other words, the integrable complex structure $j$ can be completed to a U(3) structure (and then to an SU(3) structure, as we will see), though not to a Kähler one.

This is also a good point to make some remarks about the nature of the curve $C$ that we will need later on. The concept of holomorphic curve makes sense even without an integrable complex structure; the definition is still that $(\delta+i j)_{n}^{m} \partial X^{n}=0$, where $X$ is the embedding $C$ in $\mathcal{M}$. For $j$ integrable this is the usual condition that the curve be holomorphic. But this condition makes sense even for an almost complex structure, a fact which is expressed by calling the curve pseudo-holomorphic [29]. We will often drop this prefix in the following. In many of the usual manipulations involving calibrated cycles, one never uses integrability properties for the almost complex or symplectic structures on $\mathcal{M}$. For example, it is still true that the restriction of $J$ to $C$ is its volume form $\operatorname{vol}_{C}$. Exactly in the same way, one can speak of Special Lagrangian submanifolds even without integrability (after having defined an SU(3) structure, which we will in the next section), and sometimes we will qualify them as "pseudo" to signify this.

Let us now consider $2 \rightarrow 3$ transitions. It will turn out that the results are just mirror of the ones we gave for $3 \rightarrow 2$, but in this case it is probably helpful to review them separately. After all, mirror symmetry for complex-symplectic pairs is not as well established as for Calabi-Yaus, which is one of the motivations of the present work. (Evidence so far includes mathematical insight [2, and, in the slightly more general

\footnotetext{
${ }^{5}$ In the mirror picture, a similar argument shows immediately that $d \Omega \neq 0$ on $\mathcal{W}^{\prime}$, and hence the manifold cannot be complex.
} 
context of SU(3) structure manifolds, comparisons of four-dimensional theories [32, 33] and direct SYZ computation [34].)

Suppose now we start (in the IIA theory) with a symplectic manifold $\mathcal{W}$ (whose moduli space is, as we said, modeled on $\left.H^{2}(\mathcal{M}, \mathbb{R})\right)$, and that for some value of the symplectic moduli some curves shrink. Then, it turns out that one can always replace the resulting singularities by some three-cycles, and still get a symplectic manifold (Theorem 2.7, [3]). The trick is that $T^{*} S^{3}$, the deformed conifold, is naturally symplectic, since it is a cotangent bundle. Then [3] proves that this holds even globally: there is no problem in patching together the modifications around each conifold point. One should compare this with the construction used by Hirzebruch and Reid cited above.

It is not automatic that the resulting manifold $\mathcal{W}^{\prime}$ is complex, even if $\mathcal{W}$ is complex itself. The criterion is that there should be at least one relation in homology between the collapsing curves $C_{a}$ [35, 36] (see also 37] for an interesting application). ${ }^{6}$

Let us collect the transitions considered so far in a table; we also anticipate in which string theory each transition will be relevant for us. The symmetry among these results is clear; we will not need all of them, though.

\begin{tabular}{|c|c|c|c|c|c|}
\cline { 2 - 6 } \multicolumn{1}{c|}{} & transition & keeps symplectic & keeps complex & $\Delta b_{2}$ & $\Delta b_{3}$ \\
\hline \hline IIA & $2 \rightarrow 3$ & yes $([\underline{3}, 2.7)$ & if $\sum r_{i}^{a} C_{a}=0([\underline{35},[36])$ & $N-R$ & $2 R$ \\
\hline IIB & $3 \rightarrow 2$ & if $\sum r_{i}^{a} B_{a}=0([3], 2.9)$ & yes & $R$ & $2(N-R)$ \\
\hline
\end{tabular}

Table 1: The conditions for a transition to send a complex or symplectic conifold to a complex or symplectic manifold.

\subsection{Vacua versus geometry}

We can now apply the results reviewed in the previous subsection to our vacua. Remember that in IIB we have chosen a point in moduli space in which a single three-cycle shrinks, and in IIA one in which a single curve shrinks.

From our assumptions, the singularities affect the manifold only locally (as opposed for example to the IIA case of [1], in which the quantum volume of the whole manifold

\footnotetext{
${ }^{6}$ Actually, the criterion also assumes $\mathcal{W}$ to satisfy the $\partial \bar{\partial}$-lemma, to ensure that $H^{2,1} \subset H^{3}$, which is not always true on complex non-Kähler manifolds; this assumption is trivially valid in the cases we consider, where $\mathcal{W}$ is a Calabi-Yau.
} 
is shrinking); it is hence natural to assume that the vacua of section 2 are still geometrical. Given the experience with the Calabi-Yau case, it is also natural that the brane hypermultiplet $B$ describes a surgery. But then we can use the results of the previous subsection.

In IIB, where we have shrunk a three-cycle, we now know that the manifold obtained by replacing the node with a curve will be naturally complex, but will not be symplectic, since by assumption we do not have any relations. As we have explained, the reason for this is that on the manifold $\mathcal{M}^{\prime}$ after the transition, there will be a holomorphic curve $C$ which is homologically trivial; and by Stokes, we conclude that the manifold cannot be symplectic.

Summing up, we are proposing that in IIB the vacua we are finding are given by a complex non-symplectic (and hence non-Kähler ${ }^{7}$ ) manifold. This manifold $\mathcal{M}^{\prime}$ is defined by a small resolution on the singular point of $\mathcal{M}$, and it has (see table 1 ) Betti numbers

$$
b_{2}\left(\mathcal{M}^{\prime}\right)=b_{2}(\mathcal{M}), \quad b_{3}\left(\mathcal{M}^{\prime}\right)=b_{3}(\mathcal{M})-2 .
$$

In the example described in section 2.1, when $\mathcal{M}$ is the mirror of an elliptic fibration over $\mathbb{F}_{1}, \mathcal{M}^{\prime}$ has $b_{2}=243, b_{3} / 2=3$.

In IIA, a similar reasoning lets us conjecture that the new vacua correspond to having a symplectic non-complex (and hence non-Kähler) manifold $\mathcal{W}^{\prime}$, obtained from the original Calabi-Yau $\mathcal{W}$ by replacing the node with a three-cycle. This manifold $\mathcal{W}^{\prime}$ has

$$
b_{2}\left(\mathcal{W}^{\prime}\right)=b_{2}(\mathcal{W})-1, \quad b_{3}\left(\mathcal{W}^{\prime}\right)=b_{3}(\mathcal{W})
$$

In the example from section 2.1] when $\mathcal{W}$ is an elliptic fibration over $\mathbb{F}_{1}, \mathcal{W}^{\prime}$ has $b_{2}=2$, $b_{3} / 2=244$.

Notice that these two sets of vacua are mirror by construction: we localize in IIA and in IIB to points which are mirror to each other, and in both cases we add the appropriate brane hypermultiplets to reveal new lines of vacua. What is conjectural is simply the interpretation of the vacua. We now proceed to give evidence for that conjecture.

\footnotetext{
${ }^{7}$ There might actually be, theoretically speaking, a Kähler structure on the manifold which has nothing to do with the surgery. This question is natural mathematically [3, but irrelevant physically: such a Kähler structure would be in some other branch of moduli space, far from the one we are considering, which is connected and close to the original Calabi-Yau by construction.
} 
In the IIB case, the spectrum before the transition is clearly given by $b_{3}(\mathcal{M}) / 2-1$ vector multiplets and $b_{2}(\mathcal{M})+1$ hypermultiplets (the " +1 " is the universal hypermultiplet). We have seen that the potential generated by $F_{3}$ gives mass to one of the vector multiplets, fixing it at a certain point in the complex moduli space. On the other side, the number of massless hypermultiplets remains the same. Indeed, we have added a brane hypermultiplet $B$; but this combines with the universal hypermultiplet to give only one massless direction, the one given in (2.13).

This is to be compared with the Betti numbers of the proposed $\mathcal{M}^{\prime}$ from table 1 . indeed, $b_{2}$ remains the same and $b_{3}$ changes by 2 . Since the manifold is now non-Kähler, we have to be careful in drawing conclusions: "Kähler moduli" a priori do not make sense any more, and though complex moduli are still given by $H^{2,1}$ (by Kodaira-Spencer and $K=0$ ), a priori this number is $\neq b_{3} / 2-1$, since the manifold is non-Kähler.

However, two circumstances help us. The first is that, by construction, the moduli of the manifolds we have constructed are identified with the moduli of the singular CalabiYau on which the small resolution is performed. Then, indeed we can say that there should be $b_{3}\left(\mathcal{M}^{\prime}\right) / 2-1+b_{2}\left(\mathcal{M}^{\prime}\right)$ complex geometrical moduli in total (after complexifying the moduli from $b_{2}$ with periods of the anti-symmetric tensor field appropriately, and neglecting the scalars arising from periods of RR gauge fields).

A more insightful approach exists, and will also allow us to compare low-lying massive states. Reduction on a general manifold of SU(3) structure (along with a more general class which will not concern us here) has been performed recently in [33. (Manifolds with $S U(3)$ structures and various differential conditions were also considered from the perspective of supergravity vacua, starting with [38, 39]). We have introduced a U(3) structure in the previous section as the presence on the manifold of both a complex and a symplectic structure with a compatibility condition. The almost complex structure $j$ allows us to define the bundle of $(3,0)$ forms, which is called the canonical bundle as in the integrable case. If this bundle is topologically trivial the structure reduces further to $\mathrm{SU}(3)$. The global section $\Omega$ of the canonical bundle can actually be used to define the almost complex structure by

$$
T_{\mathrm{hol}}^{*}=\left\{v_{1} \in T^{*} \mid v_{1} \wedge \bar{\Omega}=0\right\} .
$$

The integrability of the almost complex structure is then defined by $(d \Omega)_{2,2}=0$, something we will not always require. 
Let us now review the construction in 33 from our perspective. In general the results of 33 require one to know the spectrum of the Laplacian on the manifold, which is not always at hand; but in our case we have hints for the spectrum, as we will see shortly. We have seen that a $\mathrm{U}(3)$ structure, and hence also an $\mathrm{SU}(3)$ structure, defines a metric. Let us see it again: since $J \wedge \Omega=0, J$ is of type $(1,1)$, and then a metric can be defined as usual: $g_{i \bar{j}}=-i J_{i \bar{j}}$.

We can now consider the Laplacian associated to this metric. The suggestion in [33, 32] is to add some low-lying massive eigen-forms to the cohomology. Since $[\Delta, d]=0$ and $[\Delta, *]=0$, at a given mass level there will be eigen-forms of different degrees. Suppose for example $\Delta \omega_{2}=m^{2} \omega_{2}$ for a certain $m$. Then

$$
d \omega_{2} \equiv m \beta_{3}
$$

will also satisfy $\Delta \beta_{3}=m^{2} \beta_{3}$, and similarly for $\alpha_{3} \equiv * \beta_{3}$ and $\omega_{4} \equiv * \omega_{2}$. (The indices denote the degrees of the forms.) We can repeat this trick with several mass levels, even if coincident.

After having added these massive forms to the cohomology, we can use the resulting combined basis to expand $\Omega=X^{I} \alpha_{I}+\beta^{I} F_{I}$ and $J=t_{i} \omega_{i}$, formally as usual but with some of the $\alpha$ 's, $\beta$ 's and $\omega$ 's now being massive. Finally, these expansions for $\Omega$ and $J$ can be plugged into certain "universal" expressions for the Kähler prepotential $\mathcal{P}^{\alpha}$. Without fluxes (we will return on this point later) and with some dilaton factor suppressed, this looks like 33

$$
\mathcal{P}^{1}+i \mathcal{P}^{2}=\int d(B+i J) \wedge \Omega, \quad \mathcal{P}^{3}=\int\left(d C_{2}-C_{0} d B\right) \wedge \Omega
$$

Since the reader may be confused about the interpretation of the expressions $\int d(B+$ $i J) \wedge \Omega$ and $\int\left(d C_{2}-C_{0} d B\right) \wedge \Omega$ which appear above (given the ability to integrate by parts), let us pause to give some explanation. Our IIB solutions indeed correspond to complex manifolds, equipped with a preferred closed 3-form which has $d \Omega=0$. However, the $4 \mathrm{~d}$ fields which are given a mass by the gauging actually include deformations of the geometry which yield $d \Omega \neq 0$, as we discussed above. Therefore, the potential which follows from (3.8) is a nontrivial function on our field space.

Let us try to apply the KK construction just reviewed to the manifold $\mathcal{M}^{\prime}$. First of all we need some information about its spectrum. We are arguing that $\mathcal{M}^{\prime}$ is obtained 
from surgery. In [1], it is found that the spectrum of the Dirac operator changes little, in an appropriate sense, under surgery. If we assume that this result goes through after twisting the Dirac operator, we can in particular consider the Dirac operator on bispinors, also known as the signature operator, which has the same spectrum as the Laplacian. All this suggests that for very small $B$ and $g_{s}$ the spectrum on $\mathcal{M}^{\prime}$ will be very close to the one on $\mathcal{M}$. Hence there will be an eigenform of the Laplacian $\omega$ with a relatively small eigenvalue $m$ (and its partners discussed above), corresponding to the extra harmonic forms generating $H^{3}$ before the surgery. By the reasoning above, this will also give eigenforms $\alpha, \beta$ and $\tilde{\omega}$.

Expanding now $\Omega=X^{1} \alpha+\Omega_{0}, J=t^{1} \omega+J_{0}, B=b^{1} \omega+B_{0}$ and $C_{2}=c^{1} \omega+C_{20}$ (where $\Omega_{0}, J_{0}, B_{0}$ and $C_{20}$ represent the part of the expansion in cohomology) and using the relation $\int_{\mathcal{M}^{\prime}} \beta_{3} \wedge \alpha_{3}=1$, we get from (3.8):

$$
\mathcal{P}^{1}+i \mathcal{P}^{2} \sim m\left(b^{1}+i t^{1}\right) X^{1}, \quad \mathcal{P}^{3} \sim m\left(c^{1}-C_{0} b^{1}\right) X^{1}
$$

The parameter $m$ measures the non-Kählerness away from the Calabi-Yau manifold $\mathcal{M}$, and should be proportional to the vev of the brane hypermultiplet $\tilde{B}_{0}$ of $\S 2.2$. Clearly the formula is reminiscent of the quadratic dependence on the $B$ hypermultiplet in (2.12). The size of the curve $C$ is measured by $t^{1}$. Of course $\tilde{B}_{0}$ is really a function of the $t^{1}$ and universal hypermultiplets. Presumably, it and the massive hyper $\tilde{B}_{m}$ in section 2.2 are different linear combinations of the curve volume and $g_{s}$. It is even tempting to map the $\mathcal{M}$ and $\mathcal{M}^{\prime}$ variables by mapping $B$ directly to $\int_{C} J=t^{1}$, and (very reasonably) mapping the dilaton hypermultiplet on $\mathcal{M}$ directly into the one for $\mathcal{M}^{\prime}$. Indeed, the size of $C$ would then be proportional to $g_{s}$ (at least when both are small), which is consistent with both being zero at the transition point.

Fixing this would require more detailed knowledge of the map between variables. However, since the formula for the Killing prepotentials has the universal hypermultiplet in it (which can be seen from (3.9), where $C_{0}$ is mixed with other hypers and some dilaton factor is omitted in the front), it could have $\alpha^{\prime}$ corrections. Moreover, (3.8) is only valid in the supergravity regime where all the cycles are large compared with the string length. Hence an exact matching between the Killing prepotentials is lacking.

We can now attempt the following comparison between the spectrum of the vacua and the KK spectrum on the conjectural $\mathcal{M}^{\prime}$ : 
- On $\mathcal{M}$, one of the vectors, $X^{1}$, is given a mass by the gauging $\int F_{3} \wedge \Omega$. On $\mathcal{M}^{\prime}$, this vector becomes a deformation of $\Omega$ which makes it not closed, $\Omega \rightarrow \Omega+\alpha$, $\Delta \alpha=m^{2} \alpha$. In both pictures, the vacuum is at the point $X^{1}=0$. On $\mathcal{M}$, this is because we have fixed the complex modulus at the point in which $A^{1}$ shrinks. On $\mathcal{M}^{\prime}$, the manifold which is natural to propose from table 1 is complex, and hence $d \Omega=0$.

- The remaining vectors are untouched by either gauging and remain massless.

- Both for $\mathcal{M}$ and for $\mathcal{M}^{\prime}$, there are $b_{2}+1$ massless hypermultiplets.

- From the perspective of the gauged supergravity analysis on $\mathcal{M}$ there is a massive hypermultiplet too: $B$ and the universal hypermultiplet have mixed to give a massless direction, but another combination will be massive. On $\mathcal{M}^{\prime}$, there is also a massive hypermultiplet: it is some combination of $g_{s}$ and $t^{1}$, which multiplies the massive form $\omega$ (with $\Delta \omega=m^{2} \omega$ ) in the expansion of $J$. To determine the precise combination one needs better knowledge of $m\left(t^{1}, g_{s}\right)$ in (3.9).

Again, this comparison uses the fact that there is a positive eigenvalue of the Laplacian which is much smaller than the rest of the KK tower, and this fact is inspired by the work in [11.

This comparison cannot be made too precise for a number or reasons. One is, as we have already noticed, that it is hard to control the spectrum, and we had to inspire ourselves from work which seemed relevant. Another is that the KK reduction of tendimensional supergravity on the manifold $\mathcal{M}^{\prime}$ will not capture the full effective field theory precisely, as we are close (at small $B$ vevs) to a point where a geometric transition has occurred. Hence, curvatures are large in localized parts of $\mathcal{M}^{\prime}$, though the bulk of the space can be large and weakly curved. And indeed, we know that ten-dimensional type II supergravities do not allow $\mathcal{N}=2$ Minkowski vacua from non-Kähler compactification manifolds in a regime where all cycles are large enough to trust supergravity (though inclusion of further ingredients like orientifolds, which are present in string theory, can yield large radius $\mathcal{N}=2$ Minkowski vacua in this context [40]). The vacua of [1], and our own models, presumably evade this no-go theorem via stringy corrections arising in the region localized around the small resolution. Some of these corrections are captured by the local field theory analysis reviewed in $§ 2.3$, which gives us a reasonable knowledge 
of the hyper moduli space close to the singularity. It should be noted that the family of vacua we have found cannot simply disappear as one increases the expectation values of the $B$ fields and $e^{\phi}$ : the moduli space of $\mathcal{N}=2$ vacua is expected to be analytic even for the fully-fledged string theory. However, new terms in the expansion of the $\mathcal{P}^{\alpha}$ 's in terms of the $B$ hypermultiplet will deform the line; and large $g_{s}$ will make the perturbative type II description unreliable.

An issue that deserves separate treatment is the following. Why have we assumed $F_{3}=0$ in (3.8)? It would seem that the integral $\int_{B} F_{3}$ cannot simply go away. Usually, in conifold transitions (especially noncompact ones) a flux becomes a brane, as the cycle becomes contractible and surrounds a locus on which, by Gauss' law, there must be a brane. This would be the case if, in figure 1 the flux were on $A$ : this would really mean a brane on $C$. In our case, the flux is on $B$, on a chain which surrounds nothing. Without sources, and without being non-trivial in cohomology, $F_{3}$ has no choice but disappear on $\mathcal{M}^{\prime}$.

To summarize this section, we have conjectured to which manifolds the vacua found in section 2 correspond. In this way, we have also provided explicit symplectic-complex non-Kähler mirror pairs.

\section{The big picture: a space of geometries}

There are a few remarks that can be made about the type of complex and symplectic manifolds that we have just analyzed, and that suggest a more general picture. This is a speculative section, and it should be taken as such.

One of the questions which motivated us is the following. The KK reduction in [33] says that $\int d J \wedge \Omega$ encodes the gauging of the four-dimensional effective supergravity on $\mathcal{M}^{\prime}$. Hence in some appropriate sense (to be discussed below), $d J$ must be integral - one would like $\int d J \wedge \Omega$ to be expressed in terms of integral combinations of periods of $\Omega$. This is just because the allowed gauge charges in the full string theory form an integral lattice. But from existing discussions, the integral nature of $d J$ is far from evident. Though one can normalize the massive forms appropriately in such a way that the expression does give an integer, this does not distinguish between several possible values for the gauging: it is just a renormalization, not a quantization. 
Without really answering this question, we want to suggest that there must be a natural modification of cohomology that somehow encodes some of the massive eigenvalues of the Laplacian, and that has integrality built in. It will be helpful to refer again to figure 1. on $\mathcal{M}^{\prime}$ (the manifold on the right in the lower line of figure 1), we have depicted a few relevant chains, obviously in a low-dimensional analogy. What used to be called the $A$ cycle is now still a cycle, but trivial in homology, as it is bounded by a four-cycle $D$. The dual $B$ cycle, from other side, now is no longer a cycle at all, but merely a chain, its boundary being the curve $C$. This curve has already played a crucial role in showing that $\mathcal{M}^{\prime}$ cannot be symplectic.

We want to suggest that a special role is played by relative cohomology groups $H_{3}\left(\mathcal{M}^{\prime}, C\right)$ and $H_{4}\left(\mathcal{M}^{\prime}, A\right)$. Remember that relative homology is the hypercohomology of $C_{\bullet}(C) \stackrel{\iota_{C}}{\longrightarrow} C_{\bullet}\left(\mathcal{M}^{\prime}\right)$, with $C_{k}$ being chains and the map $\iota_{C}$ being the inclusion. In plain English, chains in $C_{k}\left(\mathcal{M}^{\prime}, C\right)$ are pairs of chains $\left(c_{k}, \tilde{c}_{k-1}\right) \in C_{k}\left(\mathcal{M}^{\prime}\right) \times C_{k-1}(C)$, and homology is given by considering the differential

$$
\partial\left(c_{k}, \tilde{c}_{k-1}\right)=\left(\partial c_{k}+\iota_{C}\left(\tilde{c}_{k-1}\right),-\partial \tilde{c}_{k-1}\right)
$$

So cycles in $H_{k}\left(\mathcal{M}^{\prime}, C\right)$, for example, are ordinary chains which have boundary on $C$. $B$ is precisely such a chain. A long exact sequence can be used to show that, when $C$ is a curve trivial in $H_{2}\left(\mathcal{M}^{\prime}\right)$ as is our case, $\operatorname{dim}\left(H_{3}\left(\mathcal{M}^{\prime}, C\right)\right)=\operatorname{dim}\left(H_{3}\left(\mathcal{M}^{\prime}\right)\right)+1$. So $(B, C)$ and the usual cycles generate $H_{3}\left(\mathcal{M}^{\prime}, C\right)$. Similarly, $\operatorname{dim}\left(H_{4}\left(\mathcal{M}^{\prime}, A\right)\right)=\operatorname{dim}\left(H_{4}\left(\mathcal{M}^{\prime}\right)\right)+1$, and the new generator is $(D, A)$.

Similar and dual statements are valid in cohomology. This is defined similarly as for homology: pairs $\left(\omega_{k}, \tilde{\omega}_{k-1}\right) \in \Omega^{k}\left(\mathcal{M}^{\prime}\right) \times \Omega^{k-1}(C)$, with a differential

$$
d\left(\omega_{k}, \tilde{\omega}_{k-1}\right)=\left(d \omega_{k}, \iota_{C}^{*}\left(\omega_{k}\right)-d \tilde{\omega}_{k-1}\right)
$$

A non-trivial element of $H^{3}\left(\mathcal{M}^{\prime}, C\right)$ is $\left(0, \operatorname{vol}_{C}\right)$. Since $C$ is a holomorphic curve, $\operatorname{vol}_{C}=$ $J_{\mid C} \equiv \iota_{C}^{*} J$ and hence this representative is also equivalent to $(d J, 0)$, using the differential above.

When we deform $\mathcal{M}^{\prime}$ with the scalar in the massive vector multiplet $X^{1}$, the manifold becomes non-complex, as we have shown in the previous section; but one does not require the almost complex structure to be integrable to define an appropriate notion of holomorphic curve. In fact, one might expect then that, when $d \Omega \neq 0$, which corresponds to $\mathcal{M}^{\prime}$ being non-complex, one can also choose $A$ to be SLag (as we remarked earlier, 
the definition will not really require that the almost symplectic structure be closed). ${ }^{8}$ Definitely, the logic would hold the other way around - if such a SLag $A$ can be found, $\int_{A} \Omega \neq 0$ and then, again by integration by parts, it follows that $d \Omega \neq 0$.

In our example, we expect the number of units $n_{1}$ of $F_{3}$ flux present before the transition in the IIB picture, to map to " $n_{1}$ units of $d J$ " on $\mathcal{M}^{\prime}$. The phrase in quotes has not been precisely defined, but it is reasonable to think that it is defined by some kind of intersection theory in relative homology. We will now try to make this more precise.

As we have seen, the dimension of the relative $H_{3}$ can be odd (and it is in our case), so we should not expect a pairing between $A$ and $B$ cycles within the same group. One might try nevertheless to define a pairing between chains in $H_{3}\left(\mathcal{M}^{\prime}, C\right)$ and $H_{4}\left(\mathcal{M}^{\prime}, A\right)$; it would be defined by

$$
(B, C) \cdot(D, A) \equiv \#(B \cap A)=\#(C \cap D) .
$$

In fact, if we think of another lower-dimensional analogy, in which both $A$ and $C$ are one-dimensional in a three-dimensional manifold, it is easy to see that what we have just defined is a linking number between $C$ and $A$. Indeed, $\operatorname{dim}(C)+\operatorname{dim}(A)=\operatorname{dim}\left(\mathcal{M}^{\prime}\right)-1$.

This can also be rephrased in relative cohomology. Consider a bump-form $\delta_{A}$ which is concentrated around $A$ and has only components transverse to it, and similarly for $C$. These can be defined more precisely using tubular neighborhoods and the Thom isomorphism [41. Since $A$ and $C$ are trivial in homology, we cannot quite say that these bump forms are the Poincaré duals of $A$ and $C$. But we can say that $\left(\delta_{A}, 0\right) \in H^{3}(M, C)$ is the Poincaré dual to the cycle $(D, A) \in H_{4}(M, A)$, with natural definitions for the pairing between homology and cohomology. $\delta_{A}$ is non-trivial in relative cohomology but trivial in the ordinary cohomology $H^{3}(M)$, and hence there exists an $F_{A}$ such that $d F_{A}=\delta_{A}$. Then we have

$$
\int_{\mathcal{M}^{\prime}} F_{A} \wedge \delta_{C}=\int_{C} F_{A}=\int_{B} d F_{A}=\#(C \cap D) \equiv L(A, C) .
$$

In other words, in cohomology we have $L(A, C)=\int d^{-1}\left(\delta_{A}\right) \wedge \delta_{C}$.

Suppose we have now another form $\tilde{\delta}_{A}$ which can represent the Poincaré dual (in relative cohomology) to $(D, A)$. Then we can use this other form as well to compute the

\footnotetext{
${ }^{8}$ The reader should not confuse this potential SLag, which may exist off-shell in the IIB theory, with the pseudo-SLag manifold that exists on $\mathcal{W}^{\prime}$ where $d \Omega \neq 0$ even on the $\mathcal{N}=2$ supersymmetric solutions.
} 
linking, with identical result. This is because $\left(\delta_{A}, 0\right) \sim\left(\tilde{\delta}_{A}, 0\right)$ in $H^{3}\left(\mathcal{M}^{\prime}, A\right)$ means that, by the definition of the differential above, $\delta_{A}-\tilde{\delta}_{A}=d \omega_{2}$ with $\omega_{2}$ satisfying $\iota_{C}^{*} \omega_{2}=d \tilde{\omega}_{1}$ for some form $\tilde{\omega}_{1}$ on $C$. Then

$$
\int_{\mathcal{M}^{\prime}} d^{-1}\left(\delta_{A}-\tilde{\delta}_{A}\right) \wedge \delta_{C}=\int_{\mathcal{M}^{\prime}} \omega_{2} \wedge \delta_{C}=\int_{C} \omega_{2}=\int_{C} d \tilde{\omega}_{1}=0
$$

so $L(A, C)$ does not depend on the choice of the Poincaré dual. But now, remember that $(d J, 0)$ is also a non-trivial element of $H^{3}\left(\mathcal{M}^{\prime}, C\right)$; if we normalize the volume of $C$ to 1 , it then has an equally valid claim to be called a Poincaré dual to $(D, A)$. Indeed, $\int_{(B, C)}(d J, 0) \equiv \int_{B} d J=\int_{C} J=1=(D, A) \cdot(B, C)$, and for all other cycles the result is zero. Similar reasonings apply to $d \Omega$. Then we can apply the steps above and conclude that

$$
L(A, C)=\int_{\mathcal{M}^{\prime}} d J \wedge \Omega
$$

In doing this we have normalized the volumes of $C$ and $A$ to one; if we reinstall those volumes, we get precisely that $\int d J \wedge \Omega$ is a linear function of the vectors and hypers with an integral slope.

Another point which seems to be suggesting itself is the relation between homologically trivial Special Lagrangians and holomorphic curves on one side, and massive terms in the expansion of $\Omega$ and $J$ on the other. The presence of a holomorphic but trivial curve, as we have already recalled, implies that $d J \neq 0$ : in the previous section we have seen that one actually expects that such curves are in one-to-one correspondence with massive eigenforms of the Laplacian present in the expansion of $J$ (whose coefficients represent massive fields, which vanish in vacuum). We have argued for this relation close to the transition point, and for the $\mathcal{M}^{\prime}$ that we have constructed, but it might be that this link persists in general. This would mean that inside an arbitrary SU(3) structure manifold, one would have massive fields which are naturally singled out, associated to homologically trivial holomorphic curves.

Similarly, in the IIA on $\mathcal{W}^{\prime}$, there is a 3-cycle which is (pseudo) Special Lagrangian but homologically trivial. Its presence implies that $d \Omega \neq 0$, in keeping with the fact that the IIA vacua are non-complex.

Reid's fantasy [12] involved the conjecture that by shrinking -1 curves, and then deforming, one may find a connected configuration space of complex threefolds with $K=0$. Here, we see that it is natural to extend this fantasy to include a mirror conjecture: that 
the space of symplectic non-complex manifolds with $\mathrm{SU}(3)$ structure is similarly connected, perhaps via transitions involving the contraction of (pseudo) Special Lagrangian cycles, followed by small resolutions. The specialization to -1 curves in [12] is probably mirror to the requirement that the SLags be rigid, in the sense that $b^{1}=0$.

In either IIB or IIA, we have seen that (at least close to the transition) there is a natural set of massive fields to include in the low-energy theory, associated with the classes of cycles described above. Allowing these fields to take on expectation values may allow one to move off-shell, filling out a finite-dimensional (but large) configuration space, inside which complex and symplectic manifolds would be zeros of a stringy effective potential. While finding such an $\mathcal{N}=2$ configuration space together with an appropriate potential to reveal all $\mathcal{N}=2$ vacua is clearly an ambitious goal, it may also provide a fruitful warmup problem for the more general question of characterizing the string theory "landscape" of $\mathcal{N} \leq 1$ vacua [42]

In this bigger picture, this paper is a Taylor expansion of the master potential around a corner in which the moduli space of $\mathcal{M}^{\prime}$ meets the moduli space of compactifications on $\mathcal{M}$ with RR flux.

Acknowledgments: We would like to thank P. Aspinwall, B. Florea and A. KashaniPoor for useful discussions, and I. Smith and R. Thomas for some patient explanations of their work. The authors received support from the DOE under contract DE-AC0376SF00515 and from the National Science Foundation under grant 0244728. SK was also supported by a David and Lucile Packard Foundation Fellowship for Science and Engineering.

\section{A Details about an example}

We will detail here the transition for the example mentioned in section 2.1. We will do so on the IIA side, which is the one which involves the strictest assumptions, as explained there.

The Calabi-Yau $\mathcal{W}$ is an elliptic fibration over the Hirzebruch surface $\mathbb{F}_{1}$. It is convenient to describe it as a hypersurface in a toric manifold $V$. The fan for the latter is described by the columns of the matrix

$$
\begin{array}{lllllll}
v_{1} & v_{2} & v_{3} & v_{4} & v_{5} & v_{6} & v_{7}
\end{array}
$$




$$
\left[\begin{array}{ccccccc}
0 & 0 & 0 & 1 & 0 & -1 & 0 \\
0 & 0 & 1 & 1 & -1 & 0 & 0 \\
0 & -1 & 2 & 2 & 2 & 2 & 2 \\
-1 & 0 & 3 & 3 & 3 & 3 & 3
\end{array}\right]
$$

The last five vectors lie in the same plane, determined by the last two coordinates; let us plot the first two coordinates, along with three different triangulations:
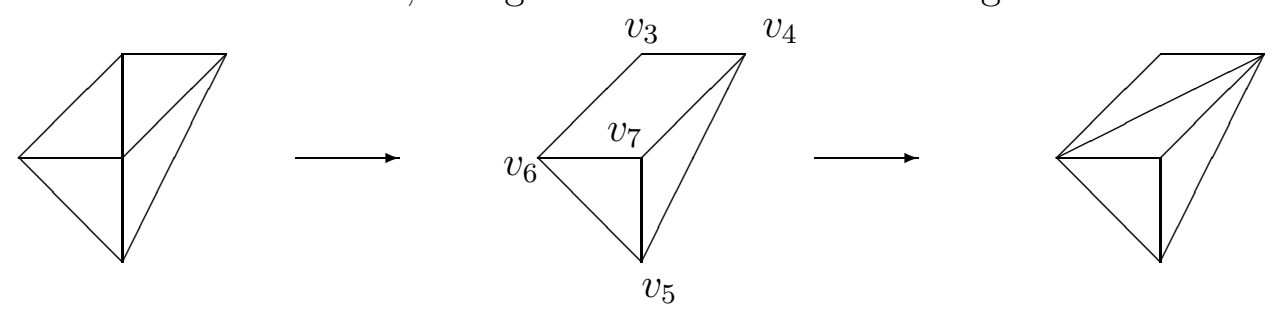

The vectors of the fan are indeed the right ones to describe the $\mathbb{F}_{1}$ base. The fan is further specified by the higher-dimensional cones in the picture, with the first triangulation really describing the elliptic fibration over $\mathbb{F}_{1}$, the last describing a space related to the first by a flop, and the middle triangulation describing the singular case. (The points have been labeled in the singular case only to avoid cluttering the picture.) We associate as usual a homogeneous coordinate $z_{i}$ to each of the $v_{i}$ 's, with charge matrix given by the (transposed) kernel of the matrix above:

$$
\left[\begin{array}{ccccccc}
0 & 0 & 3 & -2 & 1 & -2 & 0 \\
6 & 4 & 1 & 0 & 1 & 0 & 0 \\
3 & 2 & 0 & 0 & 0 & 0 & 1
\end{array}\right]
$$

From the picture we see that the flopped locus in $V$ lies at $z_{3}=z_{4}=z_{6}=z_{7}=0$. One has to check whether this locus intersects the Calabi-Yau only once. This is done by looking at the equation for $\mathcal{W} \subset V$, which for a certain point in the complex moduli space reads $z_{1}^{2}+z_{2}^{3}+z_{3}^{12} z_{4}^{18} z_{7}^{6}+z_{5}^{12} z_{6}^{6} z_{7}^{6}+z_{3}^{12} z_{6}^{18} z_{7}^{6}+z_{4}^{6} z_{5}^{12} z_{7}^{6}=0$; hence we get the singular locus $z_{1}^{2}+z_{2}^{3}=0$ on $\mathcal{W}$. Taking into account the $\mathbb{C}^{*}$ actions, this corresponds to only one point $p$ as desired. To verify that the normal bundle of the shrinking curve has charges $(-1,-1)$, one can identify the combination of the charges that keeps $p$ invariant; this action turns out to be $\left(1,1, \lambda, \lambda^{-1}, 1, \lambda^{-1}, \lambda\right), \lambda \in \mathbb{C}^{*}$, which is the right one for a conifold point. 


\section{References}

[1] J. Polchinski and A. Strominger, "New Vacua for Type II String Theory," Phys. Lett. B 388, 736 (1996) arXiv:hep-th/9510227.

[2] M. Kontsevich, "Homological algebra of mirror symmetry," Proceedings of ICM (Zürich, 1994), 120-139. Birkhäuser, Basel, 1995, alg-geom/9411018.

[3] I. Smith, R. P. Thomas, S.-T. Yau, "Symplectic conifold transitions," Jour. Diff. Geom. 62, 209 (2002) arXiv:math.SG/0209319.

[4] I. Smith, R. P. Thomas, "Symplectic surgeries from singularities," Turkish J. Math. 27, 231 (2003) arXiv:math.SG/0212213.

[5] A. Strominger, "Superstrings with torsion," Nucl. Phys. B 274, 253 (1986).

[6] M. Grana, R. Minasian, M. Petrini and A. Tomasiello, "Generalized structures of $\mathcal{N}=1$ vacua," arXiv:hep-th/0505212.

[7] N. Hitchin, Quart. J. Math. Oxford Ser. 54, 281 (2003) arXiv:math.dg/0209099.

[8] M. Grana, R. Minasian, M. Petrini and A. Tomasiello, "Supersymmetric backgrounds from generalized Calabi-Yau manifolds," JHEP 0408 (2004) 046 arXiv:hep-th/0406137.

[9] G. Dall'Agata, "On supersymmetric solutions to IIB supergravity with general fluxes," Nucl. Phys. B 695, 243 (2004) arXiv:hep-th/0403220.

[10] A. Frey, "Notes on $S U(3)$ structures in type IIB supergravity," JHEP 0406, 027 (2004) [arXiv: hep-th/0404107.

[11] Ch. Bär and M. Dahl, "Surgery and the Spectrum of the Dirac Operator," J. reine angew. Math. 552, 53-76 (2002).

[12] M. Reid, "The moduli space of $3-F o l d s$ with $K=0$ may nevertheless be irreducible," Math. Ann. 278, 329-334.

[13] For recent discussions of supersymmetric non-geometric vacua, see: J. Shelton, W. Taylor and B. Wecht, "Nongeometric flux compactifications," arXiv:hep-th/0508133, and references therein. 
[14] B. R. Greene, "String theory on Calabi-Yau manifolds," arXiv:hep-th/9702155.

[15] P. S. Aspinwall, B. R. Greene and D. R. Morrison, "Calabi-Yau moduli space, mirror manifolds and spacetime topology change in string theory," Nucl. Phys. B 416, 414 (1994) arXiv:hep-th/9309097.

[16] E. Witten, "Phases of $\mathcal{N}=2$ theories in two dimensions," Nucl. Phys. B 403, 159 (1993) arXiv:hep-th/9301042.

[17] D. R. Morrison and C. Vafa, "Compactifications of F-Theory on Calabi-Yau Threefolds - I," Nucl. Phys. B 473, 74 (1996) arXiv:hep-th/9602114.

[18] J. Louis, J. Sonnenschein, S. Theisen and S. Yankielowicz, "Non-perturbative properties of heterotic string vacua compactified on $K 3 \times T^{2}$," Nucl. Phys. B 480, 185 (1996) arXiv:hep-th/9606049.

[19] J. Michelson, "Compactifications of type IIB strings to four dimensions with nontrivial classical potential," Nucl. Phys. B 495, 127 (1997) arXiv:hep-th/9610151.

[20] See for instance: L. Andrianopoli, S. Ferrara and M. Trigiante, "Fluxes, supersymmetry breaking, and gauged supergravity," arXiv:hep-th/0307139, and references therein.

[21] N. Seiberg, "IR dynamics on branes and space-time geometry," Phys. Lett. B 384, 81 (1996) arXiv:hep-th/9606017; N. Seiberg and E. Witten, "Gauge dynamics and compactification to three dimensions," in Saclay 1996: The mathematical beauty of physics, pp. 333-366 arXiv:hep-th/9607163.

[22] N. Seiberg and S. Shenker, "Hypermultiplet moduli space and string compactification to three dimensions," Phys. Lett. B 388, 521 (1996) arXiv:hep-th/9608086.

[23] B. Greene, D. Morrison and C. Vafa, "A geometric realization of confinement," Nucl. Phys. B 481, 513 (1996) arXiv:hep-th/9608039; H. Ooguri and C. Vafa, "Summing up D instantons," Phys. Rev. Lett. 77, 3296 (1996) arXiv:hep-th/9608079.

[24] P. Candelas, P. S. Green and T. Hübsch, "Rolling Among Calabi-Yau Vacua," Nucl. Phys. B 330, 49 (1990); "Finite Distances Between Distinct Calabi-Yau Vacua: (Other Worlds Are Just Around The Corner),” Phys. Rev. Lett. 62, 1956 (1989). 
[25] B. Greene, D. Morrison and A. Strominger, "Black hole condensation and the unification of string vacua," Nucl. Phys. B 451, 109 (1995) |arXiv:hep-th/9504145.

[26] T. M. Chiang, B. R. Greene, M. Gross and Y. Kanter, "Black hole condensation and the web of Calabi-Yau manifolds," Nucl. Phys. Proc. Suppl. 46, 82 (1996) arXiv:hep-th/9511204.

[27] F. Hirzebruch, "Some examples of threefolds with trivial canonical bundle," notes by J. Werner, Max Planck Inst. preprint no.85-58, Bonn 1985.

[28] J. Moser, "On the volume elements on a manifold," Trans. Amer. Math. Soc. 120 (1965), 286-294.

[29] M. Gromov, "Pseudo-holomorphic curves in symplectic manifolds," Invent. Math. 82 (1985), 307-347.

[30] B. van Geemen and J. Werner, "New examples of threefolds with $c_{1}=0$," Math. Z. 203 (1990), 211-225.

[31] J. Werner, "Kleine Auflösungen spezieller dreidimensionaler Varietäten," Ph. D. Thesis, Bonn.

[32] S. Gurrieri, J. Louis, A. Micu and D. Waldram, "Mirror symmetry in generalized Calabi-Yau compactifications," Nucl. Phys. B 654, 61 (2003) arXiv:hep-th/0211102.

[33] M. Grana, J. Louis and D. Waldram, "Hitchin functionals in $\mathcal{N}=2$ supergravity," arXiv:hep-th/0505264.

[34] S. Fidanza, R. Minasian and A. Tomasiello, "Mirror symmetric SU(3)structure manifolds with NS fluxes," Commun. Math. Phys. 254, 401 (2005) arXiv:hep-th/0311122.

[35] R. Friedman, "Simultaneous resolution of threefold double points," Math. Ann. 274, 671 (1986).

[36] G. Tian, "Smoothing 3-folds with trivial canonical bundle and ordinary double points," in Essays on Mirror Manifolds (S.-T. Yau, ed.), Hong Kong, International Press, 458-479 (1992). 
[37] P. Lu and G. Tian, "The complex structures on connected sums of $S^{3} \times S^{3}$," in Manifolds and geometry, Cambridge University Press (1993).

[38] S. Chiossi, S. Salamon, "The intrinsic torsion of SU(3) and G2 structures," Proc. conf. Differential Geometry Valencia 2001, math.DG/0202282.

[39] J. P. Gauntlett, D. Martelli, S. Pakis and D. Waldram, "G-structures and wrapped NS5-branes," Commun. Math. Phys. 247 (2004) 421 arXiv:hep-th/0205050.

[40] S. Kachru, M. Schulz, P. Tripathy and S. Trivedi, "New supersymmetric string compactifications," JHEP 0303, 061 (2003) arXiv:hep-th/0211182.

[41] See for instance R. Bott and L. Tu, Differential Forms in Algebraic Topology, Springer (1982), pp. 65-69.

[42] R. Bousso and J. Polchinski, "Quantization of four-form fluxes and dynamical neutralization of the cosmological constant," JHEP 0006, 006 (2000) arXiv:hep-th/0004134; S.B. Giddings, S. Kachru and J. Polchinski, "Hierarchies from fluxes in string compactifications," Phys. Rev. D 66, 106006 (2002) arXiv:hep-th/0105097; A. Maloney, E. Silverstein and A. Strominger, "de Sitter space in noncritical string theory," arXiv:hep-th/0205316 S. Kachru, R. Kallosh, A. Linde and S. Trivedi, "de Sitter vacua in string theory," Phys. Rev. D 68, 046005 (2003) [arXiv: hep-th/0301240; L. Susskind, "The anthropic landscape of string theory," arXiv:hep-th/0302219, M. Douglas, "The statistics of string/M theory vacua," JHEP 0305, 046 (2003) arXiv:hep-th/0303194. 